\title{
Advanced Concepts in Waveguide Spectrometers
}

\author{
P. Cheben, J.H. Schmid, A. Bogdanov, M.L. Calvo, A. Delâge, A. Densmore, S. Janz, B. Lamontagne, \\ J. Lapointe, O. Martínez Matos, E. Post, I. Powell, J. A. Rodrigo, P. Waldron, and D.-X. Xu
}

\begin{abstract}
We present several new concepts to extend the capabilities of arrayed waveguide grating spectrometers. Strong lateral mode confinement waveguide structure in the focal plane is used to increase resolution, a Michelson-type AWG spectrometer can improve optical throughput or étendue, and we show that AWG dispersion can be magnified many times by modifying the waveguide group index.
\end{abstract}

\section{INTRODUCTION}

Waveguide spectrometers, including waveguide echelle gratings and arrayed waveguide gratings (AWGs) [1,2], perform functions such as WDM multiplexing and demultiplexing, wavelength filtering, signal routing and optical cross-connection, among others. New applications of spectrometer chips are emerging in optical interconnects, spectroscopy, metrology, chemical and biological sensing, medical instrumentation, and space-based sensing [3]. Compact waveguide spectrometer chips can potentially be made with many channels and a large spectral resolution by using waveguide platforms with high index contrast, such as silicon-on-insulator. The first SOI AWG was reported by Trinh et al. in 1997 [4]. By taking advantage of the high-index contrast of $\mathrm{Si}$ waveguides, we demonstrated a compact 8channel 200-GHz SOI AWG microspectrometer with a footprint of $5 \times 5 \mathrm{~mm}^{2}$ [5]. Using similar designs, we have subsequently developed new methods to produce polarization insensitive devices [6-9].

In this paper we review new concepts our group is exploring to further enhance the performance and extend the applications of AWG-based spectrometers, including AWG spectral resolution, light throughput (étendue), and wavelength dispersion.

\section{HIGH-RESOLUTION SOI AWG SPECTROMETER}

The wavelength resolution of a bulk optic grating spectrometer is determined, for a given grating and imaging optics, by the input slit (in a monochromator also the output slit) width. By narrowing the slit width, the resolution is

Manuscript received April 28, 2006.

P. Cheben, J.H. Schmid, A. Bodganov, J. Lapointe, A. Delâge, A. Densmore, S. Janz, B. Lamontagne, I. Powell, E. Post, P. Waldron, and D.-X. Xu are with the Institute for Microstructural Sciences, National Research Council of Canada, 1200 Montreal Rd., Ottawa, Ontario, K1A 0R6, Canada. I. Powell is with the Steacie Institute for Molecular Sciences, National Research Council of Canada. M. L. Calvo, O. Martínez Matos, and J. A. Rodrigo are with the Departamento de Óptica, Facultad de Ciencias Físicas, Universidad Complutense de Madrid, 28040 Madrid, Spain.

Corresponding author: Pavel Cheben, phone: 1-613-9931624, fax: 1-6139907656, e-mail: pavel.cheben@,nrc-cnrc.gc.ca. improved. In an AWG spectrometer, the input and output waveguide mode size at the combiner focal planes plays the analogous role to the slit width, but this mode size is limited to several microns in glass waveguide AWGs. The mode size and coupling between closely spaced waveguides also limits the maximum number of channels. In silicon however, well confined single mode waveguides, or photonic wires, can be fabricated with dimensions as small as $0.2 \mu \mathrm{m}$. This suggests that by adopting photonic wire technology into AWG design, compact spectrometer with extremely high resolution and channel count can be fabricated [10,11].

In our spectrometer design (Fig. 1), we use deeply etched slit-like $1.5 \times 0.6 \mu \mathrm{m}^{2}$ waveguides separated by $0.4 \mu \mathrm{m}$ air gaps (Fig. 1d), to couple into or accept light from the input and output combiners, respectively.
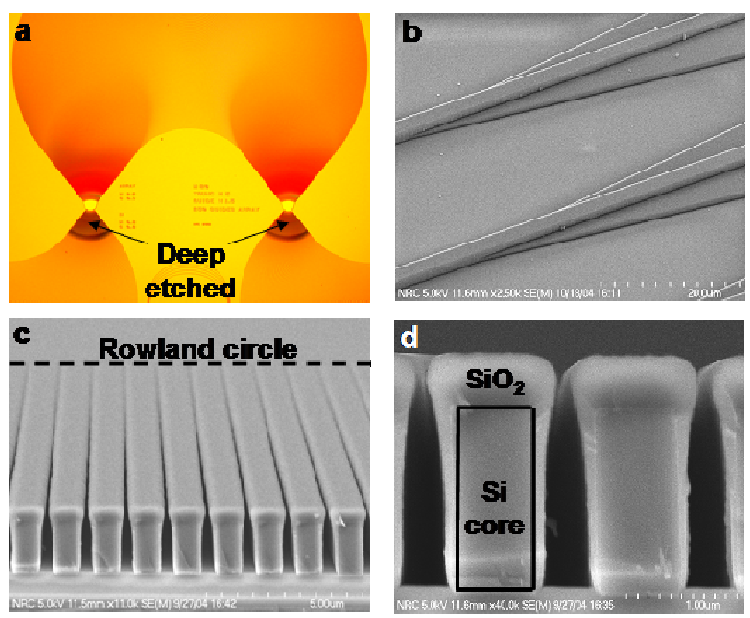

Fig. 1. a) A microphotograph of a 100-channel SOI AWG spectrometer; b) SEM image of a ridge SOI waveguide adiabatically transformed into a deepetch rectangular waveguide before joining a slab waveguide at the Rowland circle; c) Deep etched waveguides near the Rowland circle; d) detail of (c).

As a result the input mode width launched into the input combiner, and its corresponding image width projected along the focal curve (i.e. the Rowland circle) of the output combiner, are minimized. Different spectral channels are intercepted by the slit waveguides densely arrayed along the output combiner focal curve, as shown in Figs. 1c and 1d. The spectrometer has 100-channels with a wavelength resolution of $0.8 \AA$ and a total chip size of $8 \times 8 \mathrm{~mm}^{2}$. Devices were fabricated on SOI substrates with a $1.5 \mu \mathrm{m}$ thick Si waveguide layer and $1 \mu \mathrm{m}$ thick buried oxide.

The input waveguides and array waveguides are conventional partially etched ridge waveguides. However, near the Rowland circles, the ridge waveguide are adiabatically transformed down to $0.6 \mu \mathrm{m}$ wide slit 
waveguides deep etched down to the buried oxide. The ridge waveguides and two-level adiabatic mode converters shown in Fig. $1 \mathrm{~b}$ are fabricated using a self-aligned two-step e-beam patterning and plasma etching process.

Fig. 2 shows the measured spectra for several adjacent spectrometer channels. The adjacent and non-adjacent crosstalk is $10 \mathrm{~dB}$ or better. The measured channel spacing is $2 \AA$ (25 GHz at $1550 \mathrm{~nm})$, allowing to resolve spectral lines separated by $\sim 1 \AA$. The waveguide scattering loss $(\sim 15 \mathrm{~dB})$ is the largest contribution to the insertion loss, and the probable cause of the relatively high cross-talk. Both will be improved as our unique two-level fabrication process is optimized.

This is the highest resolution reported up to date for an SOI AWG. The size advantage of this device is obvious. Previously reported $25-\mathrm{GHz}$ silica AWGs occupy a 4-inch wafer $[1,13]$, two orders of magnitude larger than the $8 \times 8$ $\mathrm{mm}^{2}$ footprint of our spectrometer. This principle can also be used in low index contrast platforms (e.g. silica-on-silica) by etching air trenches [12] to increase adiabatically the mode confinement near the Rowland circles.

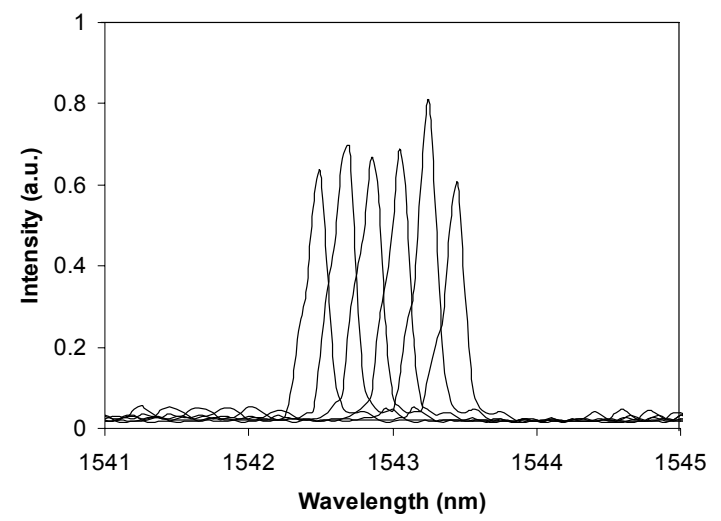

Fig. 2 Measured spectra for several adjacent channels of the SOI AWG spectrometer. The light intensity is shown in linear scale. TM polarization.

\section{FOURIER-TRANSFORM MICHELSON-TYPE AWG}

The need for narrow input waveguide apertures to achieve a high resolution can be avoided by arranging the AWG in a Michelson configuration, which allows for a wider input aperture, a property known as étendue (light throughput) advantage. To explore this benefit, we proposed [14] a Fourier transform waveguide spectrometer using two interleaved reflective AWGs in a Michelson configuration, as shown in Fig 3a. Light emerging from an input waveguide (IW) of width $w$ is coupled through a slab waveguide combiner (SWC) into two interleaved reflective arrayed waveguide gratings $\mathrm{AWG}_{1}$ and $\mathrm{AWG}_{2}$ of interference orders $m$ and $-m$, respectively. By interleaving the two AWGs, beam splitting and combining are achieved in a simple way (wavefront sharing), eliminating the need for a dedicated beam splitter/combiner element. The light propagates through each individual waveguide towards the truncation mirrors that reverse the light propagation direction. As the wavelength changes from the Littrow wavelength, the two wavefronts originating from the waveguide arrays $\mathrm{AWG}_{1}$ and $\mathrm{AWG}_{2}$ tilt in the opposite direction according to the $\mathrm{AWG}$ dispersion relation. This results in Fizeau interference fringes with a wavelength-dependent period in the slab waveguide combiner where the two wavefronts overlap. The interference fringes are brought to a convenient location (image curve, IC) by imaging optics (mirror, M), where the interferogram $I(x)$ is sampled by receiver waveguides (RW). Monochromatic light of wavelength $\lambda$ produces a sinusoidal interferogram, whereas for arbitrary input spectral distribution $B(\lambda)$, the spectrum $B(\lambda)$ can be retrieved by Fourier transformation of the interferogram $I(x)$.
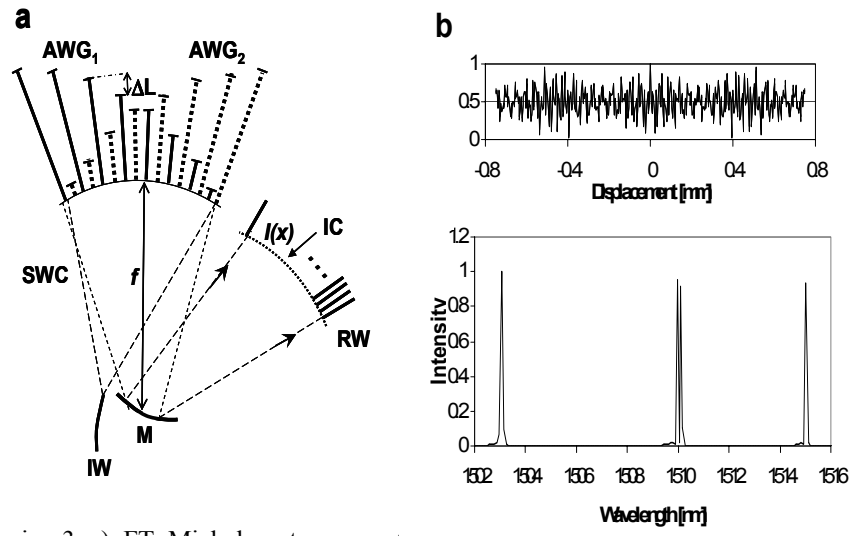

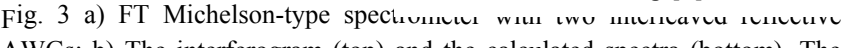
AWGs; b) The interferogram (top) and the calculated spectra (bottom). The light source comprises four monochromatic lines at wavelengths of $1503 \mathrm{~nm}$, $1510 \mathrm{~nm}, 1510.1 \mathrm{~nm}$, and $1515 \mathrm{~nm}$.

A Fourier transform AWG device has been designed for the silicon-on-insulator (SOI) platform [14]. Fig. 3b shows the simulated interferogram (top) for an input waveguide of width $w=40 \mu \mathrm{m}$, and the spectra (bottom) obtained by Fourier transformation of the interferogram. The two peaks near 1510 $\mathrm{nm}$, which are separated by $0.1 \mathrm{~nm}$, are well resolved for this large waveguide aperture. This is a significant increase in the aperture size compared to the grating based microspectrometers, as the latter would require, for a similar resolution and interference order, an input waveguide width of $\sim 1 \mu \mathrm{m}$. There are two important advantages of this new type of device. First, as a Michelson-type interferometer it allows a larger input aperture size as compared to diffraction grating based instruments, providing an increased light throughput. Second, unlike most of the state-of-the-art FT spectrometers that require scanning mirrors, our FT AWG is a static device and gives a spectral resolution equivalent to a conventional FT spectrometer with a mirror scanning range of $\sim 10 \mathrm{~mm}$. This scanning range cannot be achieved with the current MEMS technology.

\section{AWG DISPERSION ENHANCEMENT EFFECT}

We have reported [15] that AWG dispersion and resolution can be increased by modifying the group index of the waveguide array. When the waveguide group index is modified within the triangular region of the waveguide array (Fig. 4a), the AWG dispersion equation becomes:

$\sin \theta=\frac{\left(\lambda-\lambda_{c}\right)\left(M+M^{\prime}\right)}{n_{e f f, s} \Lambda}=\frac{\left(\lambda-\lambda_{c}\right)\left(n_{g} \Delta L+\Delta n_{g} \Delta L_{g}\right)}{\lambda_{c} n_{e f f, s} \Lambda}$, 
where $M=m n_{g} / n_{\text {eff }}$ is the interference order of a conventional AWG with length increment $\Delta L$ between the adjacent waveguides, $M^{\prime}=m \Delta n_{g} / \Delta n_{\text {eff }}$ is the interference order enhancement factor due to group index modification, $\Delta L_{g}$ is the length difference between the adjacent waveguides in the triangular section (Fig. 4a) with modified group index, and $\Delta n_{g}$ and $\Delta n_{\text {eff }}$ are respective differences in group and effective indices between the modified and unmodified waveguide sections. The index $s$ in (1) refers to the slab waveguide combiner.
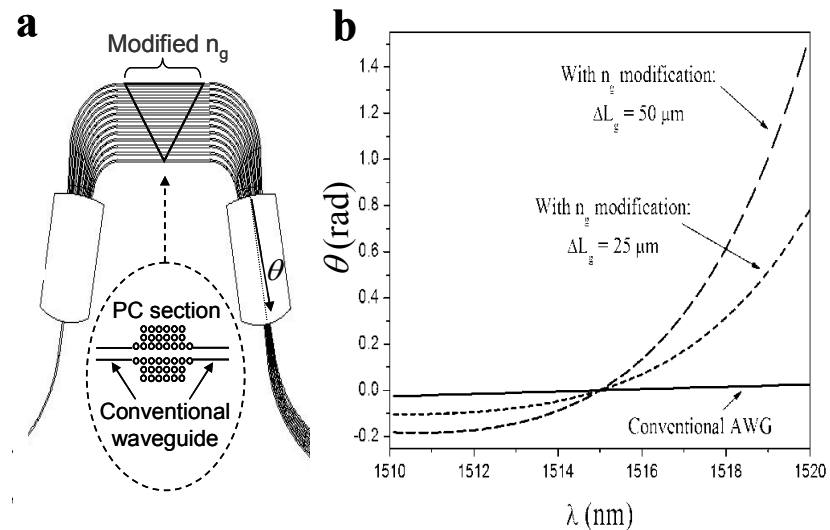

Fig. 4. a) AWG with a triangular dispersion enhancing region with large group index; b) Dispersion enhancement calculated for a triangular region with photonic crystal (PC) waveguides as indicated in (a).

Very large $\Delta n_{g}$ and hence a large dispersion enhancement can be obtained over a limited wavelength range near the edge of the stop band of various structures, including gratings, resonators, or photonic crystals. A 300-fold increase in group index has been reported by Vlasov et al. in SOI photonic crystal waveguides [16]. Fig. $4 \mathrm{~b}$ shows the calculated dispersion enhancement effect for an SOI AWG [15] using the group index measurement by Notomi et al. [17].

\section{CONCLUSIONS}

We have reviewed our progress in the development of compact waveguide spectrometers. The example of the compact 100-channel SOI AWG spectrometer suggests that high-resolution spectroscopic applications may be performed at the chip level in the foreseeable future. The second example, the Fourier transform Michelson-type AWG, has an advantage of large étendue, and as such it can be relevant for spectral analysis of light from diffuse and non-collimated sources, and for improving the spectrometer light gathering capability particularly when the light sources are weak, such as in Raman spectroscopy. Finally, we show how using a section with a large group index in the waveguide array markedly enhances the wavelength dispersion of an AWG.

\section{ACKNOWLEDGEMENT}

Part of this work has been supported by the NRC Genome and Health Initiative.

\section{REFERENCES}

1. P. Cheben, "Wavelength dispersive planar waveguide devices: echelle gratings and arrayed waveguide gratings," in Optical Waveguides: from Theory to Applied Technologies, M. L. Calvo and V. Lakshminarayanan, Eds., Taylor and Francis, London, 2006, Chap. 5.

2. S. Janz, Silicon-based waveguide technology for wavelength division multiplexing, in Silicon Photonics, L. Pavesi and D. J. Lockwood, Eds., Berlin, Springer-Verlag, 2004, chapter 10.

3. S. Janz, P. Cheben, A. Delâge, B. Lamontagne, M.-J. Picard, D.-X. Xu, K.P. Yap, and W.N. Ye, "Microphotonics: Current challenges and applications," in Frontiers in planar lightwave circuit technology, design, simulation, and fabrication, S. Janz, J. Čtyroký, and S. Tanev, Eds., Nato Science Series II Mathematics, Physics and Chemistry, vol. 216, pp. 1-38, Springer, Berlin, 2006.

4. P.D. Trinh, S. Yegnanarayanan, F. Coppinger, and B. Jalali, "Silicon-oninsulator (SOI) phased-array wavelength multi/demultiplexer with extremely low-polarization sensitivity," IEEE Photon. Technol. Lett., vol. 9, pp. 940-941, 1997.

5. M.R.T. Pearson, A. Bezinger, A. Delâge, J. W. Fraser, S. Janz, P.E. Jessop, and D.-X. Xu, "Arrayed waveguide grating demultiplexers in silicon-on-insulator," in Silicon-based Optoelectronics II, SPIE Proc. vol. 3953, pp. 11-18, 2000.

6. P. Cheben, D.-X. Xu, S. Janz, and A. Delâge, "Scaling down photonic waveguide devices on the SOI platform," in VLSI Circuits and Systems, J.F. Lopez, J.A. Montiel-Nelson, D. Pavlidis, Eds., SPIE Proc. vol. 5117, pp. 147-156, 2003.

7. P. Cheben, A. Delâge, L. Erickson, S. Janz, and D.-X. Xu, "Polarization compensation in silicon-on-insulator arrayed waveguide grating devices," in Silicon-based and hybrid optoelectronics III, SPIE Proc. vol. 4293, pp. 15-22, 2001.

8. P. Cheben, D.-X. Xu, S. Janz, A. Delâge, and D. Dalacu, "Birefringence compensation in silicon-on-insulator planar waveguide demultiplexers using a buried oxide layer," in Optoelectronic Integration on Silicon, D. J. Robbins and G. E. Jabbour, Eds., SPIE Proc. vol. 4997, pp. 181-189, 2003.

9. D.-X. Xu, P. Cheben, D. Dalacu, A. Delâge, S. Janz, B. Lamontagne, M.J. Picard, and W.N. Ye, "Eliminating the birefringence in silicon-oninsulator ridge waveguides by use of cladding stress." Optics Letters, vol. 29, pp. 2384-2386, 2004.

10. P. Cheben, A. Bogdanov, A. Delâge, S. Janz, B. Lamontagne, M. J. Picard, E. Post, and D-X. Xu, "A 100-channel near-infrared SOI waveguide microspectromer: Design and fabrication challenges," in Optoelectronics Devices and Integration, SPIE Proc. vol. 5644, pp. 103110, 2005.

11. A. Delâge, S. Bidnyk, P. Cheben, K. Dossou, S. Janz, B. Lamontagne, M. Packirisamy, and D.-X. Xu, "Recent developments in integrated spectrometers, in Proc. of the 6th International Conference on Transparent Optical Networks (ICTON), Wroclaw, Poland, vol.2, pp. 78-83, July 2004.

12. M. Popović, K. Wada, S. Akiyama, H.A. Haus, J. Michel, "Air trenches for sharp silica waveguide bends," J. Lightwave Technol., vol. 20, pp. 1762-1772, 2002.

13. K. Takada, M. Abe, T. Shibata, and K. Okamoto, "Tandem multi/ demultiplexer covering the S-, C-, and L-bands using arrayed waveguide grating with Gaussian passband as primary filter," IEEE Photon. Technol. Lett., vol. 14, pp. 648-650, 2002.

14. P. Cheben, I. Powell, S. Janz, and D.-X. Xu, "Wavelength-dispersive device based on a Fourier-transform Michelson-type arrayed waveguide grating," Optics Letters, vol. 30, pp. 1824-1826, 2005.

15. O. Martínez, M.L. Calvo, P. Cheben, S. Janz, J.A. Rodrigo, D.-X. Xu, and A. Delâge, "Arrayed waveguide grating based on group index modification," Journal of Lightwave Technology, vol. 24, pp. 1551-1557, 2006.

16. Y.A. Vlasov, M. O'Boyle, H.F. Hamann, and S.J. McNab, "Active control of slow light on a chip with photonic crystal waveguides," Nature, vol. 438, pp. 65-69, 2005.

17. M. Notomi, K. Yamada, A. Shinya, J. Takahashi, C. Takahashi, and I. Yokohama, "Extremely large group-velocity dispersion of line-defect waveguides in photonic crystal slab," Phys. Review Lett., vol. 87, 253902-1, 2001 